Research Paper

\title{
Changes and Significance of SYP and GAP-43 Expression in the Hippocampus of $\mathrm{ClH}$ Rats
}

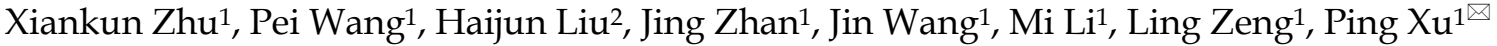 \\ 1. Department of Neurology, Affiliated Hospital of Zunyi Medical University, No. 149 Dalian Road, Zunyi, Guizhou, China, 563003 \\ 2. Key Laboratory of Basic Pharmacology of Ministry of Education, Zunyi Medical University, Zunyi, Guizhou, China, 563003 \\ $\bowtie$ Corresponding author: xuping527@vip.sina.com (Ping Xu) , Tel: +86 851 28608641, Fax: +86 85128608641 \\ ( ) Ivyspring International Publisher. This is an open access article distributed under the terms of the Creative Commons Attribution (CC BY-NC) license \\ (https:// creativecommons.org/licenses/by-nc/4.0/). See http://ivyspring.com/terms for full terms and conditions.
}

Received: 2018.07.07; Accepted: 2018.12.17; Published: 2019.01.29

\begin{abstract}
Synaptophysin (SYP) and growth-associated binding protein 43 (GAP-43) have been shown to be closely related to hippocampal synaptic plasticity in recent years. They are important molecular markers associated with synaptic plasticity. However, the role of SYP and GAP-43 in chronic intermittent hypoxic injury of the central nervous system needs to be further clarified. In this study, 25 adult male sprague dawley (SD) rats were randomly divided into a normal control group (CON) and a chronic intermittent hypoxia group $(\mathrm{ClH})$ with four time points as follows: $1 \mathrm{~W}, 2 \mathrm{~W}, 3 \mathrm{~W}$, and $4 \mathrm{~W}$. The behavioural changes (primarily learning and memory abilities) were observed by the Morris water maze in each group, consisting of 5 rats per group.The localization of SYP and GAP-43 in hippocampal CAI neurons was observed, and the expression of SYP and GAP-43 in the hippocampus was detected by Western blotting. The results showed that the mean oxygen saturation of the tail artery in $\mathrm{ClH}$ rats was less than that in normal rats $(P<0.05)$. The escape latency of $\mathrm{ClH}$ rats was longer than that of normal rats, and the number of space exploration platform crossings was less than that of normal rats. SYP-positive stained cells were yellow or brown and were mainly expressed on the cell membrane, while the GAP-43-positive staining was brown and was mainly expressed on the cell membrane and in the cytoplasm. The expression of SYP in plasma decreased gradually at the four time points for the $\mathrm{ClH}$ group $(\mathrm{P}<0.05)$, while the expression of GAP-43 in the CIH IW group increased $(\mathrm{P}<0.05)$ and decreased gradually in the $\mathrm{CIH}$ $2 \mathrm{~W}, \mathrm{ClH} 3 \mathrm{~W}$ and $\mathrm{ClH} 4 \mathrm{~W}$ groups $(\mathrm{P}<0.05)$.
\end{abstract}

Key words: Obstructive sleep apnoea syndrome; chronic intermittent hypoxia; synaptophysin; growth-associated binding protein 43 ; cognitive impairment

\section{Introduction}

Obstructive sleep apnoea syndrome (OSAS) is currently the most common clinical disease of sleep-disordered breathing, which is characterized mainly by sleep deprivation, hypoxemia, hypercapnia, and $\mathrm{pH}$ disorder [1]. In addition to causing sleep disorder and increasing the incidence of hypertension and cardiovascular and cerebrovascular diseases, the disease significantly affects the cognitive function of patients, which is mainly characterized by an overall cognitive decline, including functional impairments in aspects of attention, memory, orientation, calculation, and execution [2]. Cognitive impairment is associated with cell apoptosis and synaptic plasticity. In the nervous system, hippocampal tissues are mainly responsible for learning and memory, and they may also facilitate long-term learning and memory as well as acousto-optic and taste-related events [3]. A substantial number of studies have reported that OSAS may lead to inattention, decreased efficiency at work, a decline in executive ability and other cognitive impairments in patients; and many experiments have shown that OSAS rats also exhibit pathological damage to their hippocampus [4,5]. However, the pathogenesis of cognitive impairment caused by OSAS remains unclear, which is one of the 
causes of dissatisfactory clinical curative effects. Therefore, the determination of the cognitive functions of OSAS rats and an in-depth investigation of the expression of related proteins in hippocampal tissues will contribute to the provision of novel ideas for clinical diagnosis and treatment. Currently, the mechanism of OSAS has been widely studied because $\mathrm{CIH}$ animal models can better simulate the pathogenesis of OSAS [6].

It has been reported that the cognitive impairment caused by OSAS may be related to hypoxia, sleep fragmentation, neurotransmitters, inflammatory mediators, and changes in related brain areas, which may cause the loss of synapses, thus impairing cognitive function [7,8]. Whether information transmission, processing and neuronal storage can proceed smoothly depends on whether the structure and function of synapses are intact. Synaptic plasticity is the ability to change synaptic structure and function to adapt to environmental changes and information storage. It plays a vital role in the development of the nervous system learning and memory. Changes in synaptic plasticity may cause changes in synaptic structure, altering not only the number and size of synapses but also the number of presynaptic vesicles [9]. Synaptophysin (SYP) is a type of calcium binding glycoprotein widely distributed in the vesicular membrane of the nerve presynaptic membrane. SYP weighs $38 \mathrm{kDa}$, releases $\mathrm{Ca}^{2+}$-dependent neurotransmitters and is involved in the introduction and recirculation of synaptic vesicles and in the induction and dimension of long-term potentiation (LTP). It is regarded as a specific marker protein of presynaptic terminals. Its expression accurately reflects the synaptic density, distribution area and functional state and thus reflects plasticity [10]. Studies have indicated that OSAS synapses experience structural and functional changes, and some scholars believe that the cognitive impairment associated with OSAS is a cause of Alzheimer's disease [11]. Decreases in learning and memory abilities in neonatal rats after ischaemia and hypoxia are related to ultrastructural changes in hippocampal neurons and the number and density of synapses [12]. $\mathrm{Wu}$ et al. ${ }^{[13]}$ indicated that hypoxic and ischaemic neonatal rats exhibit chronic learning and memory impairment, and the progressive decreases in hippocampal neurons and SYP expression comprise one potential mechanism. However, the protein expression of SYP has not been clearly elucidated in the course of OSAS-induced cognitive dysfunction.

GAP-43 (growth-associated binding protein 43) is a growth-cone-rich neural tissue-specific phosphoprotein that is closely related to synaptic plasticity, axonal regeneration, and neural development [14]. It is composed of 226 amino acids and is a highly hydrophilic protein that is easily phosphorylated by PKC. Phosphorylated GAP-43 is involved in the regulation of growth and plasticity by regulating cytoskeletal components [15]. In the rat central nervous system, GAP-43 expression peaks in the rat hippocampus and neocortex two weeks after birth, which is consistent with synapse formation and GAP-43 mRNA and protein expression in most brain regions. The level of expression is significantly reduced. A large amount of expression has been observed in the marginal zone and the junctional zone, indicating that plasticity associated with long-term memory was identified ${ }^{[16]}$. Overexpression of GAP-43 and PKC genes significantly enhanced the long-term enhancement and learning ability of transgenic mice, which was associated with LTP and memory, suggesting that GAP-43 is involved in the regulation of learning and memory. Studies have shown that in the early stage of the nervous system development, GAP-43 content is increased in the neuronal cytoplasm. As the brain develops, GAP-43 expression gradually decreases; however, when brain injury occurs, its expression increases and gradually decreases with increasing time of injury repair and gradually decreases in the injured area [17]. Therefore, GAP-43 is regarded as an important marker for investigating nerve growth, development and repair. Kawasaki et al. ${ }^{[18]}$ have reported that the expression of GAP-43 in rat hippocampal neurons and the cerebral cortex is increased after hypoxia, which suggests that after hypoxia injury, the nervous system initiates a self-protection mechanism and repairs damaged neurons. Continually increasing GAP-43 expression is one approach to repair nerve damage. A previous study [19] indicated that the expression of GAP-43 in the hippocampus of an ischaemia reperfusion group began to increase at 24 hours after reperfusion and peaked at day 7 . The difference between the day 14 and sham operation groups was statistically significant, which indicates that the increase in GAP-43 expression may be related to synaptic remodelling and regeneration of neurons, which comprises the endogenous compensatory mechanism of neurons after cerebral ischaemia. Researchers have reported that hippocampal neurons in rats of the epileptic model rats with acute, intermittent and chronic recurrent brain injuries have high expression levels of GAP-43. These findings suggest that increased GAP-43 expression in the central nervous system may be involved in the mechanism underlying the repair of plasticity damage. While GAP-43 expression is closely related to injury repair, the mechanism by which it changes in the OSAS rat model remains unknown. 
Based on these previous studies, a rat model was constructed using the $\mathrm{CIH}$ method to simulate OSAS. The Morris water maze was used to dynamically assess behavioural changes. Immunohistochemistry and immunoblotting were used to detect the expression of SYP and GAP-43 in the rat hippocampus before and after $\mathrm{CIH}$ to investigate the molecular biological mechanism of OSAS and cognitive dysfunction, and to provide a new experimental basis for clinical diagnosis and treatment.

\section{Materials and methods}

1.1 CIH Rats: A total of 25 adult male SD rats weighed approximately 170-190 grams and were purchased from Daping Hospital Laboratory Animal Center of Third Military Medical University (License No. SCXK (Chongqing) 2015-0005). After being fed in the same environment (temperature: $24 \pm 2^{\circ} \mathrm{C}$, humidity: 60-85\%) with natural circadian rhythm light, the mice were randomly grouped into a normal control group (control, CON) or chronic intermittent hypoxia groups $(\mathrm{CIH}) 1 \mathrm{~W}, 2 \mathrm{~W}, 3 \mathrm{~W}$, or $4 \mathrm{~W} ; 5$ rats were assigned to each group. A animal hypoxia-oxygen enrichment device(patent number: ZL2015 20485613.9) was used to fill the hypoxic chamber with nitrogen. Rats were placed in the hypoxic chamber to induce hypoxia for $90 \mathrm{~s}$. The lowest oxygen concentration in the hypoxic chamber was maintained at $(8.0 \pm 0.5 \%){ }^{[20]}$ after each rat was replaced in the chamber. Oxygen (oxygen concentration of approximately $21 \%$ ) was pumped into the hypoxic chamber again after $90 \mathrm{~s}$, forming an intermittent hypoxic process, for a complete cycle of 3 min, This cycle was repeated between the hours of 08:30-16:30 every day, for a total of approximately 8 hours of hypoxia induction per day for 4 weeks. During the experiment, fasting was forbidden, and the oxygen concentration in the cabin was measured and regulated by an intelligent oxygen concentration control detector that was equipped to be chamer. The $\mathrm{CIH}$ rat model was determined according to previously published literature reported by researchers locally and abroad, where the standards [21] of an OSAS rat model are evaluated as follows:

1. OSAS symptoms, such as abnormal breathing movements in the chest and abdomen and snoring are observable;

2. if airway obstruction occurs, the model is considered successful even without the observance of OSAS-related symptoms; and

3. changes in the pathophysiology, histology and pathology related to OSAS, such as blood oxygen saturation, oxygen partial pressure changes, and anatomic abnormalities of the airway are observable.
Table I: OSAS disease severity scoring

\begin{tabular}{lll}
\hline Degree & $\mathrm{AHI}(\mathrm{sub} / \mathrm{h})$ & Lowest $\mathrm{Sp0}_{2}$ \\
\hline Mild & $5 \sim 15$ & $85 \sim 90$ \\
Moderate & $16 \sim 30$ & $80 \sim 84$ \\
Severe & $>30$ & $<80$ \\
\hline
\end{tabular}

1.2 Morris water maze: After the OSAS rat model was successfully established, a behaviour test was conducted for each group of rats. The Morris water maze mainly consists of a place navigation test and a spatial probe test. On the day before the test, rats from all groups were placed in a pool and allowed to swim for 2 min to observe their swimming abilities and rats that could not swim were excluded from the study. The place navigation test was conducted by dividing the pool into four quadrants, placing the rats in the quadrant farthest from the platform, randomly selecting entry points in the four quadrants, and then placing the rats into the water. The time was recorded from rat entry until the rat found the platform, which was indicated as the incubation period. If a rat failed to find the platform in $2 \mathrm{~min}$, it was guided to the platform and allowed to rest for 10-20 s. The place navigation test was conducted for 5 days, and the test was conducted at the same time each day. The spatial probe test was conducted one day after the place navigation test by removing the platform, but maintaining the other conditions. The rats were placed in the water in the centre of each quadrant (facing the wall) from the second quadrant in a clockwise manner.Then, rats were allowed to swim for $2 \mathrm{~min}$, and the effective number of crossings of the original platform were recorded. After the test, we statistically analysed the obtained data.

1.3 Tissue preparation: For immunohistochemistry, $4 \%$ paraformaldehyde was used to fix hippocampal tissue. After the antibody was added and incubated for 24 hours, the tissue sections were analysed by optical microscopy. For Western blotting, the hippocampus was quickly removed from ice and frozen at $-80^{\circ} \mathrm{C}$ in liquid nitrogen for further use.

1.4 Western blotting (WB): Briefly, proteins were extracted according to the manufacturer's instructions (Jiangsu Kaiji, China). An SDS-PAGE gel (5\% layer gel) was used for separation by gel electrophoresis with a $10 \%$ gel, and the total protein lysates $(20 \mu \mathrm{L}$ per lane) were separated by electrophoresis. Then, the separated lysates were transferred to a polyvinylidene fluoride (PVDF) membrane (BIO-RAD). The Polyvinylidene fluoride membrane was incubated with 5\% BSA for approximately 2 hours at room temperature to prevent non-specific binding. Then, the membrane was incubated overnight with rabbit anti-SYP (1:15000, Abcam, Cambridge, MA, USA), rabbit 
anti-GAP-43 antibody (1:10000, Abcam, Cambridge, MA, USA), and mouse anti-beta-tubulin antibody (1:3000, ProteinTech, USA). Next, a horseradish-labelled goat anti-rabbit IgG $(\mathrm{H}+\mathrm{L})$ (1:5000, Beijing Zhongshanjinqiao Biotechnology Co., Ltd.) secondary antibody was incubated with the PVDF membrane. After incubation, TBST solution was used to wash the membrane in a shaker three times for $10 \mathrm{~min}$ per wash. Finally, the luminescent agent $(A: B=1: 1)$ was fully exposed to the PVDF film using a BIO-Chemi DocTM Touch Imagine System.

1.5 Immunohistochemistry: The reagents and equipment uesd for immunohistochemistry included rabbit anti-SYP antibody (Abcom), rabbit anti-GAP-43 antibody (Abcom), mouse anti-beta-tubulin antibody (ProteinTech), marker protein (ProteinTech), 0.01 MPBS dry powder (Beijing Soleboro Technology Co., Ltd.); horseradish peroxidase-labelled goat anti-rabbit IgG anti-GAP-43 secondary antibody $(\mathrm{H}+\mathrm{L})$ (Beijing Zhongshanjinqiao Biotechnology Co., Ltd.) , horseradish peroxidase-labelled goat anti-mouse IgG anti-GAP43 secondary antibody $(\mathrm{H}+\mathrm{L})$ (Beijing Zhongshanjinqiao Biotechnology Co., Ltd.), and an inverted microscope (Japan OLYMPUS).

\subsection{Statistical analysis:}

Experimental data are expressed as the mean \pm standard deviation $(\bar{x} \pm \mathrm{s})$. The data were statistically analysed using SPSS 18.0 statistical analysis software. A paired t-test was used to determine the average blood oxygen saturation of the rat tail artery. Repeated measures analysis of variance was performed on the Morris water maze data, and one-way analysis of variance was performed on the WB data from different groups at the different time points. $\quad P<0.05$ indicates statistically significant differences.

\section{Results}

\subsection{Establishment of the OSAS rat model}

In this study, a closed hypoxic chamber was used to simulate an animal model with $\mathrm{CIH}$ that conformed to OSAS pathophysiological characteristics. In the process, the rats in the $\mathrm{CIH}$ group were observed to be dispirited, unresponsive, and subject to hypoxia symptoms and signs, such as waking up as a result of laboured breathing, lifting of the head to breathe, and deepened and quickened abdominal breathing. As shown by the detection of the blood oxygen saturation levels in the rat tail artery in the $\mathrm{CIH}$ group, the average blood oxygen saturation of the rat tail artery (mean $\mathrm{Sp}_{2}, \mathrm{MSp}_{2}$ ) of rats in the $\mathrm{CIH}$ group that were placed in the hypoxic chamber was $69.72 \pm 1.68 \%$, and the fluctuation range of the overall $\mathrm{SpO}_{2}$ was $60-72 \%$. When the rats were placed back in the air environment (reoxygenation interphase), the $\mathrm{MSp}_{2}$ was $96.38 \pm 1.61 \%$, its overall fluctuation range was $93-100 \%$, and the low amount of oxygen before and after $\mathrm{MSp}_{2}$ decreased by $>4 \%$, which was statistically significant $(P<0.05)$. The rat model conformed to the pathophysiological characteristics of OSAS and was successfully established (Figure 1).

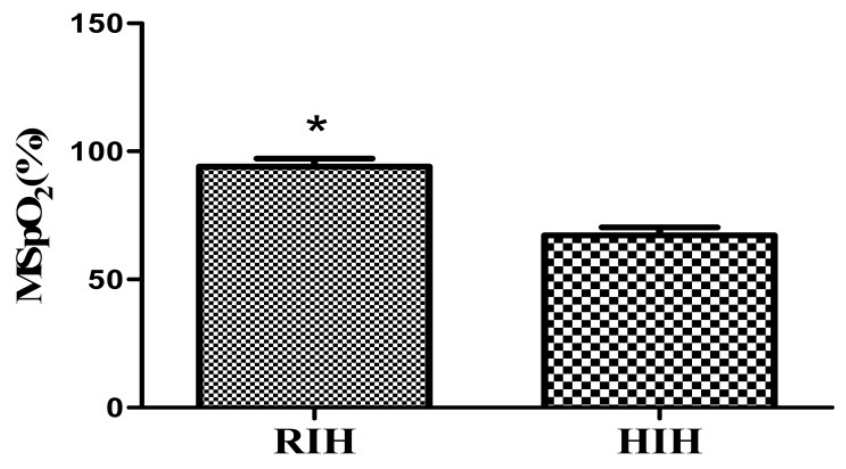

Figure 1: Changes in $\mathrm{MSpo}_{2}$ levels in the rat tail artery during the reoxygenation interphase (RIH) and hypoxia interphase (HIH) in OSAS model rats. Note: Compared with hypoxia interphase, ${ }^{*} P<0.05$.

\subsection{Animal behaviour test with the Morris water maze}

A behaviour test was conducted for the rats in each group. In the positioning voyage test, compared with the normal group, the escape latency of rats in the $\mathrm{CIH} 1 \mathrm{~W}$ group was prolonged; however, the difference was not significant $(P>0.05)$. In the $\mathrm{CIH} 2 \mathrm{~W}$, $\mathrm{CIH} 3 \mathrm{~W}$ and $\mathrm{CIH} 4 \mathrm{~W}$ groups, the escape latency was prolonged, and the difference was significant $(P<0.05)$. In the space exploration test, the number of platform crossings among the rats in the normal group was $5.03 \pm 1.51$, whereas the platform crossing numbers in the $\mathrm{CIH} 1 \mathrm{~W}, \mathrm{CIH} 2 \mathrm{~W}, \mathrm{CIH} 3 \mathrm{~W}$, and $\mathrm{CIH}$ $4 \mathrm{~W}$ groups were $4.95 \pm 1.05,3.76 \pm 0.65,2.58 \pm 0.34$, and $1.89 \pm 0.28$, respectively. Compared with the normal group, the difference in the $\mathrm{CIH} 1 \mathrm{~W}$ group was not significant $(P>0.05)$, whereas those in the $\mathrm{CIH} 2 \mathrm{~W}$, $\mathrm{CIH} 3 \mathrm{~W}$, and $\mathrm{CIH} 4 \mathrm{~W}$ groups were significant $(P<0.05)$ (Figure 2 and Figure 3 ).

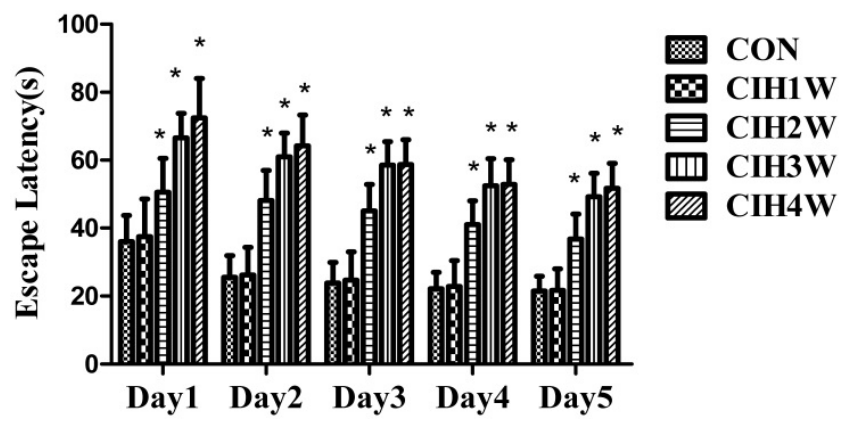

Figure 2: Changes in the escape latency of $\mathrm{CIH}$ model rats at different time points. Note: Compared with CON, $* P<0.05$. 


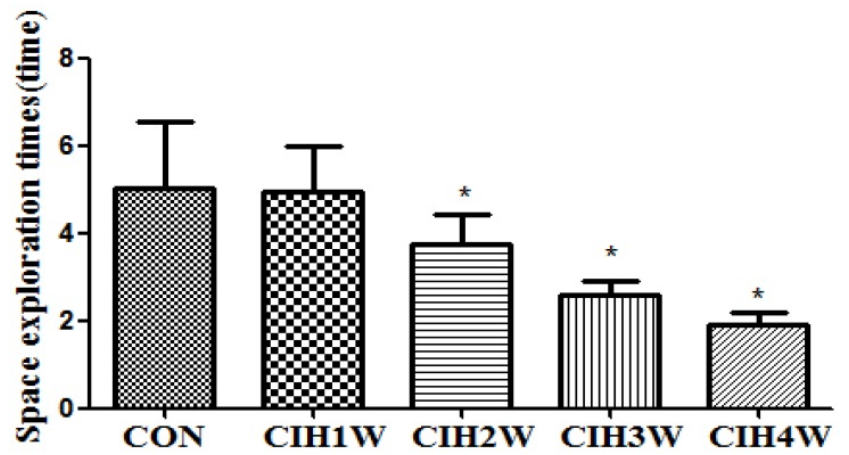

Figure 3: Changes in the number of platform crossings of $\mathrm{ClH}$ model rats. Note: Compared with $\mathrm{CON},{ }^{*} P<0.05$.

\subsection{Changes in SYP protein expression in the hippocampal tissues of $\mathrm{CIH}$ model rats}

The protein expression levels of SYP and the corresponding internal parameter ( $\beta$-tubulin) in the hippocampal tissues of all groups were analysed via Western blotting, which showed that the expression of SYP and $\beta$-tubulin was detected in the hippocampal tissues of the rats in all groups. By analysing the IOD vales with IPP software, the IOD ratio of SYP and $\beta$-tubulin (SYP/ $\beta$-tubulin) in the normal control group was determined to be 2.8532 \pm 0.0761 . In the $\mathrm{CIH} 1 \mathrm{~W}, \mathrm{CIH} 2 \mathrm{~W}, \mathrm{CIH} 3 \mathrm{~W}$, and $\mathrm{CIH} 4 \mathrm{~W}$ groups, the IOD ratios were $2.3253 \pm 0.0526$, $1.8622 \pm 0.0516,1.5386 \pm 0.0293$, and 1.12015 \pm 0.0102 , respectively, which were significantly different from that in the control group (Figure 4).

A

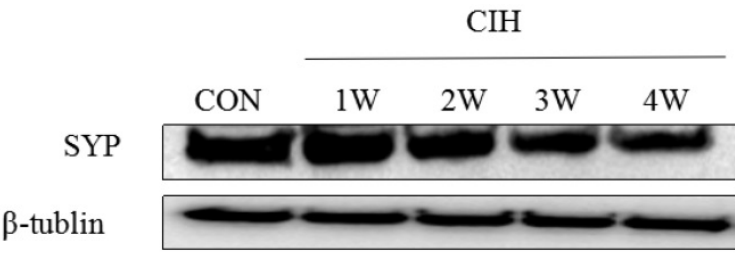

B

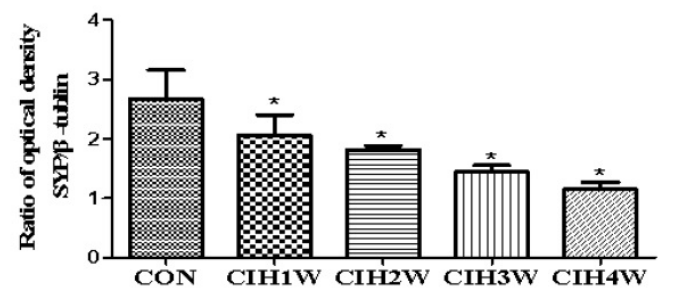

Figure 4: Changes in SYP protein expression in the hippocampal tissues of CIH model rats. Note: (A) SYP and $\beta$-tubulin protein representative strip; (B) Relative SYP protein expression; compared with CON, ${ }^{*} P<0.05$.

\subsection{Changes in GAP-43 protein expression in the hippocampal tissues of $\mathrm{ClH}$ model rats}

The protein expression levels of GAP-43 and the corresponding internal parameter ( $\beta$-tubulin) in the hippocampal tissues of all groups were analysed via Western blotting, which showed that the expression levels of GAP-43 and $\beta$-tubulin were detectable in the hippocampal tissues of the rats in all groups. By analysing the IOD values with IPP software, the IOD ratio of GAP-43 to $\beta$-tubulin (GAP-43/ $\beta$-tubulin) in the normal control group was $2.0948 \pm 0.0450$. The IOD ratios in the $\mathrm{CIH} 1 \mathrm{~W}, \mathrm{CIH} 2 \mathrm{~W}, \mathrm{CIH} 3 \mathrm{~W}, \mathrm{CIH} 4 \mathrm{~W}$ groups were 2.5070 $\pm 0.0254,1.8161 \pm 0.0358$, $1.6104 \pm 0.0337$, and $1.3803 \pm 0.0398$, respectively, which were significantly different from that in the normal group $(P<0.05)$

A

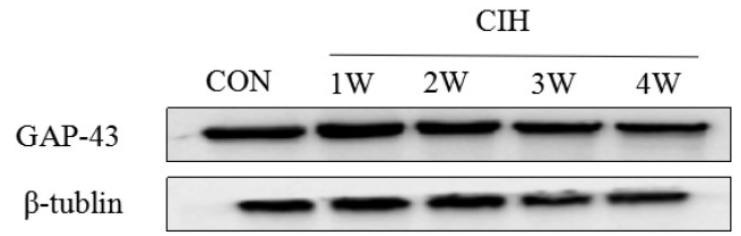

B

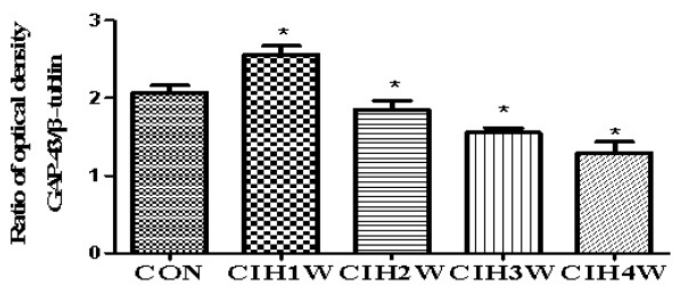

Figure 5: Changes in the protein expression of GAP-43 in the hippocampal tissues of CIH model rats. Note: (A) GAP-43 and $\beta$-tubulin protein representative strip; (B) Relative GAP-43 protein expression; compared with $\mathrm{CON},{ }^{*} \mathrm{P}<0.05$.

\subsection{Expression orientations of SYP and GAP-43 in neurocytes}

Orientations of SYP and GAP-43 expression in the hippocampal tissues of rats in the normal and $\mathrm{CIH}$ $4 \mathrm{~W}$ groups were evaluated using immunohistochemistry analysis. SYP was expressed on the neurocyte membrane of hippocampal CA1 in rats in the two groups, and the expression was lower in the $\mathrm{CIH} 4 \mathrm{~W}$ group than in the normal control group. GAP-43 was expressed on the neurocyte membrane and in the cytoplasm of hippocampal CA1 in rats, the expression in the $\mathrm{CIH} 4 \mathrm{~W}$ group was decreased compared with that in the normal control group (Figure 6).

\section{Discussion}

OSAS is characterized by a disturbance of the pharyngeal dilatation muscle. Its primary pathophysiological characteristic is $\mathrm{CIH}$ during sleep, and it is closely related to neurocognitive dysfunction, cardiovascular and cerebrovascular events, metabolic disorders and other diseases. It has been recognized as a very common public health problem [22]. Currently, the apnoea-hypopnea index (AHI) and nocturnal oxygen saturation are the two most 
commonly used clinical indicators to measure the severity of OSAS [23].

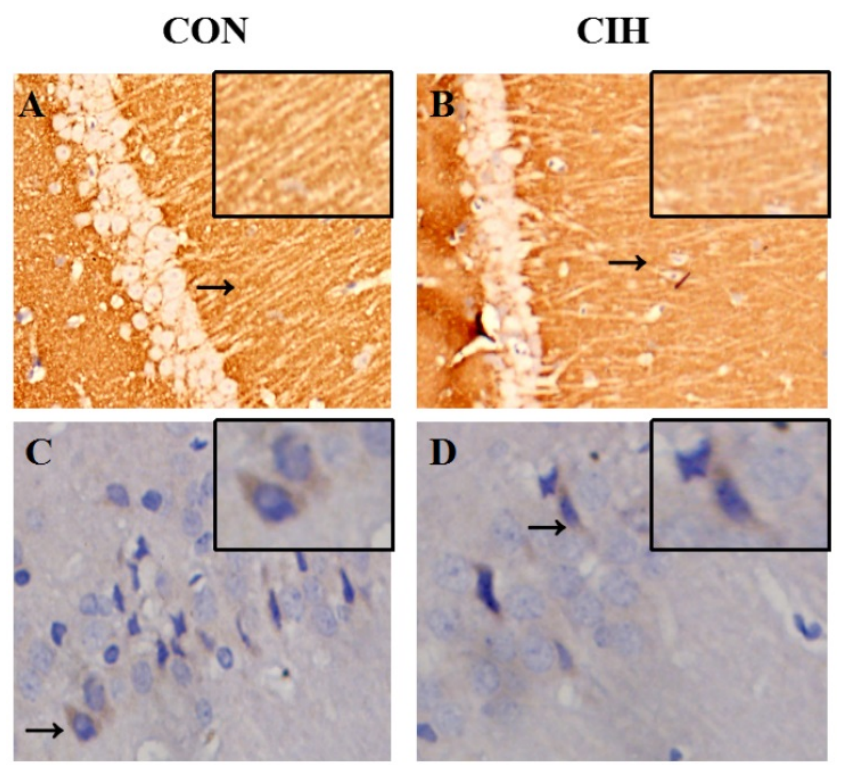

Figure 6: Sites of SYP and GAP-43 expression in the hippocampal CAl of rats in each group determined by immunohistochemical staining (40xmagnification). Note: $A$ and $B$ refer to SYP expression, and $C$ and $D$ refer to GAP-43 expression

In recent years, with increasing global attention to OSAS, there have been an increasing number of basic studies on OSAS. Therefore, the successful establishment of animal models has become an important method of evaluating the pathophysiological mechanisms, clinical features, comorbidities, new treatment methods and other aspects of OSAS. However, until now, there has been no uniform standard for the establishment of animal models of OSAS either locally or abroad. Previously, the pathological features of OSAS have been simulated by altering the upper airway or by mechanically blocking the airway. However, due to invasive operations and difficulty in controlling the severity of airway obstruction, this modelling approach is complicated, time-consuming. The success rate is also extremely low [24]. An improved $\mathrm{CIH}$ modelling method was first reported by Fletcher EC and colleagues in 1992 [25]. This method is easy to regulate and can induce different degrees of OSAS, and it also mimics important OSAS-related symptoms, such as intermittent hypoxia, lethargy, arousal and anxiety. In addition, numerous studies have shown that $\mathrm{CIH}$ can induce changes in brain function and function of rodents in the brain, such as damage to synaptic plasticity, increased apoptosis in the hippocampus and cortical neurons, and nervous system oxidative stress. The basis of brain pathological processes, such as arterial blood flow reduction and neuroinflammation, are closely related to OSAS cognitive dysfunction. Therefore, $\mathrm{CIH}$ modelling methods are widely used by a wide variety of researchers to study of OSAS mechanisms [26].

This model has been improved by Julian et al [27]. where the OSAS model is established by $\mathrm{CIH}$. This approach involves reducing the oxygen concentration in a closed chamber and placing experimental animals into the hypoxic chamber intermittently. Cyclic intermittent hypoxia is induced to mimic the pathological features of OSAS. In this study, rats were intermittently placed into the hypoxic chamber for 3 min per cycle, and intermittent hypoxia was induced for 8 hours every day for 4 weeks. Upon establishing the model, the $\mathrm{MSpO}_{2}$ was detected in rat tails. Compared with the normal control group, the $\mathrm{MSp}_{2}$ of the rats in the $\mathrm{CIH}$ group decreased by $>4 \%$, and during the experiment, the rats were observed to have wakefulness, head-up breathing, and deepening of abdominal breathing. The OSAS criteria were satisfied after observing hypoxic symptoms and signs, an apnoea index of 20 times/h, a lowest $\mathrm{MSp}_{2}$ of $<80 \%$, and a good simulation of the pathophysiological characteristics of OSAS, which indicated successful modelling.

The Morris water maze is widely used in neurocognitive foundational research. The test procedure mainly includes two components as follows: the positioning navigation test and the space exploration test ${ }^{[28]}$. In this experiment, the Morris water maze was used to detect the spatial learning and memory ability of rats to determine whether OSAS rats had cognitive dysfunction, and to simulate OSAS cognitive impairment in the animal models. By conducting a 5-day experiment on each group of rats, in the navigation test, it was found that after three days of training, the escape latency of each group of rats was significantly shortened, but it became stable on the days 4 and 5. The experimental rats had improved learning ability and formed a stable understanding and cognition of the spatial environment of the water maze. Compared with the normal group, the experimental rats in the $\mathrm{CIH} 1 \mathrm{~W}$ group had a shorter escape latency and crossed the platform fewer times, but there was no statistically significant difference, indicating that the time of hypoxia had not caused cognitive functional impairment and indicated that the rats were lacking in cognitive impairment. The oxygen tolerance was improved. From $\mathrm{CIH} 2 \mathrm{~W}$ to $\mathrm{CIH} 4 \mathrm{~W}$, the escape latency of rats gradually increased, and the number of platforms crossings was significantly less than that of the normal control group, indicating that the memory extraction and spatial association abilities of rats exposed to a $\mathrm{CIH}$ environment were impaired. 
Cognitive function is mainly manifested in attention, memory, learning ability and executive function, and plays an important role in the daily life of humans. In patients with moderate to severe OSAS, cognitive dysfunction leads to decreased work efficiency and memory loss, which seriously affects their daily life. The hippocampus is an important part of the central nervous system and plays a vital role in cognitive functions such as learning and memory, one of the most sensitive areas to hypoxia ${ }^{[29]}$. The synaptic plasticity of the hippocampus is the neural basis of learning and memory, and synaptic plasticity changes in the hippocampus are directly related to cognitive function. Synapse are important structures for the transmission of information between neurons and the formation and remodelling of neural circuits. Whether neuronal information can be transmitted, processed and stored smoothly depends on the integrity of synaptic structure and function. Synaptophysin (SYP) is a specific protein on the synaptic vesicle membrane. It consists of 307 amino acids, including four transmembrane regions. Both the amino terminus and the carboxy terminus are exposed to the cytoplasm, and the hydroxyl terminus can be combined with $\mathrm{Ca}^{2+}$, one of the synaptic vesicle calcium-binding proteins, phosphorylated by tyrosine kinase and passed through with $\mathrm{N}$-methyl-D-aspartate receptor (NMDAR) and a-amino-3-hydroxyl. The 5-methyl-4 isoxazole receptor (AMPAR) combines to form a signalling complex involved in synaptic signalling and induces the formation of long-term potentiation in the hippocampus [30]. In hippocampal neurons, SYP binds to the NMDAR subtype and anchors it to specific parts of the presynaptic membrane, which increases stability and thus affects synaptic plasticity. In addition, SYP binds to related proteins in the NMDA receptor signalling pathway and plays an important role in the regulation of synaptic states and the maintenance of synaptic structures. Studies have confirmed that SYP is closely related to the formation of synaptic plasticity in the hippocampus and is an important molecular marker associated with synaptic plasticity. $\mathrm{CIH}$ is an important pathophysiological feature of OSAS. Most studies suggest that OSAS leads to cognitive dysfunction and $\mathrm{CIH}$ leads to hippocampal structural and functional damage, especially synaptic damage in this area [31]. SYP is rich in dendritic spines, and its density and distribution can indirectly reflect the number and distribution of synapses in the body, which is closely related to cognitive function. In the early stage of neuronal development, SYP is mainly distributed in the nucleus of neurons. As the nerves mature, SYP eventually moves to the protrusions to form a point-like distribution pattern, suggesting that SYP is closely related to the formation and maturation of synapses. While $\mathrm{CIH}$ can cause hippocampal neuron death leading to denervation of axons, at this stage, hippocampal vertebral cells can prolong the spinous processes and reach new synapses at the terminals of adjacent spinous processes, while SYP is a stable extension of spinous processes. Essential synaptic proteins make hippocampal synaptic reconstruction more effective. Additionally, SYP can regulate synaptic plasticity by affecting the synaptic structure and neurotransmitter release. SYP is involved in the release of $\mathrm{Ca}^{2+}$-dependent neurotransmitters and the transport of vesicles, whose expression levels indirectly reflect the integrity and functional status of synaptic structures. The increase in SYP indicates an increase in synaptic number, synaptic transmission function, and synaptic plasticity. Therefore, damage and the number of synaptic functions are positively correlated with the degree of cognitive impairment. A previous study has shown that hypoxia can promote the activation of neuroglia and then produce excessive amounts of cellular inflammatory factors, free radicals, nitrogen oxides, mitochondrial dysfunction, etc., causing synaptic function and structural damage [32]. In transgenic mice with Alzheimer's disease, the loss of long-term SYP can aggravate the memory function of the mice, and an improvement in cognitive function is accompanied by an increase in the expression of SYP in the hippocampus. In this study, immunohistochemistry was used. It was found that SYP was highly expressed in the hippocampus of normal rats but was low in the hippocampus of the $\mathrm{CIH} 4 \mathrm{~W}$ group and was mainly localized on the membrane, indicating that chronic intermittent hypoxia leads to a decrease in SYP expression. The expression of SYP in the hippocampus at different timepoints after $\mathrm{CIH}$ was induced in rats was detected by Western blotting. It was found that SYP expression decreased with increasing $\mathrm{CIH}$ time, and the cognitive function of experimental rats also decreased, indicating a certain correlation between the two. $\mathrm{CIH}$ caused a decrease in the expression of SYP in the rat hippocampus. Combined with the behavioural results of experimental rats, it was suggested that the decrease in SYP expression may be one of the causes of cognitive dysfunction in OSAS rats.

GAP-43 is a neural tissue-specific phosphoprotein closely related to neurodevelopment and synaptic remodelling. It is abundantly expressed in the hippocampus of the nervous system, suggesting that it is associated with long-term learning and memory. When the nervous system is damaged, the neurons around the injury site are compensated by lateral branch germination and reactive axonal regeneration. The GAP-43 expresssion 
level in these areas increases with increasing damage repair time, and then gradually decreases. Therefore, the expression level of GAP-43 is considered to be the preferred probe for studying neural plasticity such as nerve regeneration and repair [33]. As previously mentioned, in the early post-injury of the nervous system, the increased expression of GAP-43 is a protective compensatory response of the body to the injury and has an intrinsic protective effect on the body. This concept has also been shown in other acute and chronic injury experiments in the hippocampus. Currently, the regulation of GAP-43 expression is theoretically conducive to the establishment of synaptic connections, and can improve the brain function of rats to some extent, and compensate for brain damage caused by $\mathrm{CIH}$. In this study, an immunohistochemical method showed that GAP-43 was highly expressed in the cytoplasm and membrane of the hippocampus of normal rats but exhibited low expression in the hippocampus of the $\mathrm{CIH} 4 \mathrm{~W}$ group, indicating that hypoxia caused decreased GAP-43 expression that was determined by Western blotting. The expression of GAP-43 in the hippocampus increased at different timepoints after $\mathrm{CIH}$ induction in the experimental rats. Compared with the normal control group, the expression of GAP-43 increased in $\mathrm{CIH} 1 \mathrm{~W}$, and then gradually decreased, indicating that $\mathrm{CIH}$ can cause the expression of GAP-43 in the hippocampus of rats to change and the increase in $\mathrm{CIH}$ 1W group is related to the events involved in hypoxia, including mossy fibre sprouting and synaptic remodelling, until the establishment of complete synaptic connections, where the GAP-43 expression level suddenly decreased, consistent with the results of Kawasaki T and others. The body or cells produce a series of adaptive responses in the early stage of hypoxia to maintain oxygen homeostasis, especially chronic intermittent hypoxia, which mainly affects gene expression and increases protein expression in the early stage. In addition, the characteristic $\mathrm{CIH}$ process of OSAS is similar to ischaemia-reperfusion, repeated hypoxia and reoxygenation, producing excessive oxygen free radicals, destroying the balance between the oxidation system and the antioxidant system, and causing oxidative stress. The body produces oxidative damage, such as cell membrane lipid peroxidation and denaturation of intracellular proteins, ultimately leading to cell death, tissue damage, and decreased expression of various intracellular proteins. Oxidative stress also induces apoptosis by activating the mitogen-activated protein kinase pathway, nuclear factor $\mathrm{\kappa B}$ pathway, and p53 pathway [34]. The brain has the highest oxygen consumption in the body, contains a large amount of polyunsaturated fatty acids, and is more susceptible to oxygen free radical attack than other tissues, and neurons can only supply energy by consuming glucose through aerobic metabolism: therefore for high concentrations of ROS, the brain is very sensitive to damage. Combining these data with the behavioural results of experimental rats, we concluded that $\mathrm{CIH} 1 \mathrm{~W}$ rats did not have significant cognitive impairment, whereas $\mathrm{CIH} 2 \mathrm{~W}, \mathrm{CIH} 3 \mathrm{~W}$ and $\mathrm{CIH} 4 \mathrm{~W}$ rats exhibited cognitive impairment. Therefore, GAP-43 expression is a crucial factor in OSAS. Decreased GAP-43 expression in rat hippocampal tissue may be one of the causes of cognitive decline. However, subsequent experiments are required for further investigation of specific associated mechanisms.

\section{Acknowledgements}

This research was supported by the National Natural Science Foundation of China (81660194).The present study was also supported by the Key Laboratory of Basic Pharmacology of Ministry of Education, Zunyi Medical University, Zunyi, Guizhou, China, 563003. We thank all partners and staff who helped us in the process of this study.

\section{Competing Interests}

The authors have declared that no competing interest exists.

\section{References}

[1] Benbir G, Karadeniz D. A pilot study of the effects of non-invasive mechanical ventilation on the prognosis of is chemic cerebrovascular events in patients with obstructive sleep apnea syndrome. J NeurolSci. 2012;33: 811-818.

[2] Torabi-Nami M, Mehrabi S, Borhani-Haghighi A,et al. Withstanding the obstructive sleep apnea syndrome at the expense of arousal instability, altered cerebral autoregulation and neurocognitive decline. J Inter Neurosci. 2015;14:169-193.

[3] Kielb SA, Ancoli-Israel S, Rebok GW, et al. Cognition in obstructive sleep apnea-hypopnea syndrome (OSAS): current clinical knowledge and the impact of treatment. J Neuromol Med. 2012;14:180-193.

[4] Dusak A, Ursavas A, Hakyemez B, et al. Correlation between hippocampal volume and excessive daytime sleepiness in obstructive sleep apnea syndrome. J Pharm Sci. 2013;17: 1198-1204.

[5] Deng Y, Yuan X, Guo XL, et al. Efficacy of atorvastatin on hippocampal neuronal damage caused by chronic intermittent hypoxia: Involving TLR4 and its downstream signaling pathway. J Respir Physiol Neurobiol. 2015;218:57-63.

[6] Badran M, Ayas N,Laher I. Insights into Obstructive Sleep apnea research. J Sleep Med. 2014;15:485-495

[7] Gildeh N, Drakatos P, Higgins S, et al. Emerging co-morbidities of obstructive Sleep apnea: cognition, kidney disease, and cancer. J Thorac Dis. 2016;8:901-917.

[8] Ferini-Strambi L, Lombardi G E, Marelli S, et al. Neurological Deficits in Obstructive Sleep Apnea. J Curr Treat Option Neurol. 2017; 19: 16.

[9] Klemann C J, Roubos E W. The gray area between synapse structure and function-Gray's synapse types I and II revisited. J Synapse. 2011;65:1222-1230.

[10] Song SH, Augustine GJ. Synapsin Isoforms and Synaptic Vesicle Trafficking. J Mol Cells. 2015;38:936-940.

[11] Daulatzai M A. Cerebral hypoperfusion and glucose hypometabolism: Key pathophysiological modulators promote neurodegeneration, cognitive impairment, and Alzheimer's disease. J Neurosci Res. 2017;95:943-972.

[12] Lal C, Strange C, Bachman D. Neurocognitive impairment in obstructive sleep Apnea. J Chest. 2012;141:1601-1610.

[13] $\mathrm{Wu} \mathrm{J} \mathrm{,} \mathrm{Li} \mathrm{P} \mathrm{,} \mathrm{Wu} \mathrm{X.} \mathrm{The} \mathrm{effect} \mathrm{of} \mathrm{chronic} \mathrm{intermittent} \mathrm{hypoxia} \mathrm{on} \mathrm{respiratory}$ sensitivity to morphine in rats. J Sleep Breath. 2017;21: 227-233.

[14] H Su JY, Stein SA, Xu X M. Temporal and Spatial distribution of growth-associated molecules and astroglial cells in the rat corticospinal tract during development. J Neurosci Res. 2010;80: 330-340. 
[15] HE Q, Dent EW. Modulation of actin filament behavior by GAP-43 (Neuromodulin) is dependent on the phosphonylation status of serine 41, the protein kinase C site. J Neurosci. 2013; 17:1515-1524.

[16] Kathryn R, William S, Damian S. McAninch, Snezana Stefanovic, et al. hnRNP-Q1 represses nascent axon growth in cortical neurons by inhibiting Gap-43 mRNA translation. J Mol Biol Cell. 2016; 27: 518-534.

[17] Hulo S, Alberi S, Laux T, et al. A point mutant of GAP-43 induces enhanced short-term and long-term hippocampal plasticity. J Eur Neurosci. 2015;15: 1976-1982.

[18] Kawasaki T, Nishio T, Kawaguchi S,et al. Spationtemporal distribution of GAP-43 in the developing rat Spinal cord: a histo-logical and quantitative immunofluorescence study. J Neuro-Sci Res. 2001;39:347-358.

[19] Crestini A, Piscopo P, Malvezzi Campeggi L, et al. Proteic marker of hypoxic-ischemic damage. J Ann Ist Super Sanita. 2001; 37: 581-591.

[20] Soukhova-O'Hare G K, Roberts A M, Gozal D. Impaired control of renal sympathetic nerve activity following neonatal intermittent hypoxia in rats. J Neuro-sci Letters. 2006; 399: 181-185.

[21] Farre R, Rotger M, Montserrat J M, et al. Collapsible upper airway segment to study the obstructive sleep apnea/hypopnea syndrom in rats. J Respir Physiol Neurobiol. 2003;5: 2-3.

[22] Natsios G, Pastaka C, Vavougios G, et al. Age, Body Mass Index, and Daytime and Nocturnal Hypoxia as Predictors of Hypertension in Patients With Obstructive Sleep Apnea. J Hypertens. 2016;18:146-152.

[23] Gonzalez C, Yubero S, Gomez-Niño MA, et al. Some reflections on intermittent hypoxia. Does it constitute the translational niche for carotid body chemoreceptor researchers? J Adv Exp Med Biol. 2012;758: 333-342.

[24] Zoccal D B , Machado B H . Sympathetic overactivity coupled with active expiration in rats submitted to chronic intermittent hypoxia. J Respir Physiol Neurobiol. 2010;174: 98-101.

[25] Fletcher E C, Lesske J, Behm R, et al. Carotid chemoreceptors, systemic blood pressure, and chronic episodic hypoxia mimicking sleep apnea. J Appl Physiol. 1992;72: 1978-1984.

[26] Chopra S, Polotsky V Y, Jun J C. Sleep Apnea Research in Animals. Past, Present, and Future. J Respir Cell Mol Biol. 2016; 54:299.

[27] Julian G S, Oliveira R W D, Perry J C, et al. Validation of Housekeeping Genes in the Brains of Rats Submitted to Chronic Intermittent Hypoxia, a Sleep Apnea Model. J Plos One. 2014; 9: e109902.

[28] Morris R G M. Spatial Localization Does Not Require the Presence of Local Cues. J Learn and Motiv. 1981;12: 239-260.

[29] Xu L H, Xie H, Shi Z H, et al. Critical Role of Endoplasmic Reticulum Stress in Chronic Intermittent Hypoxia-Induced Deficits in Synaptic Plasticity and Long-Term Memory. J Antioxid Redox Signal. 2015; 23: 695.

[30] Lisman J. Glutamatergic synapses are structurally and biochemically complex because of multiple plasticity processes:Long-term potentiation, long-term depression, short-term potentiation and scaling. J Philos Trans R Soc Lond B Biol Sci. 2017;372:1715

[31] Rao S K, Ross J M, Harrison F E, et al. Differential proteomic and behavioral effects of long-term voluntary exercise in wild-type and APP-overexpressing transgenics. J Neurobiol Dis. 2015;78:45-55

[32] Jackman K A, Zhou P, Faraco G, et al. Dichotomous effects of chronic intermittent hypoxia on focal cerebral ischemic injury. J Stroke. 2014:45:1460-1467.

[33] Ceber M, Sener U, Mihmanli A, et al. The relationship between changes in the expression of growth associated protein -43 and functional recovery of the injured inferior alveolar nerve following transection without repair in adult rats. J Craniomaxillofac Surg. 2015;43:1906-1913.

[34] Pietraforte D, Malorni W. Focusing at the double-edged sword of redox imbalance:Signal for cell survival or for cell death? J Antioxid Redox Signal. 2014:52-55. 